\title{
Transatlantica
}

Revue d'études américaines. American Studies Journal

\section{The Plot Against America : l'auteur, le lecteur et le complot.}

\section{Arnaud Schmitt}

\section{(2) OpenEdition}

1 Journals

\section{Édition électronique}

URL : http://journals.openedition.org/transatlantica/1832

DOI : $10.4000 /$ transatlantica.1832

ISSN : $1765-2766$

Éditeur

AFEA

\section{Référence électronique}

Arnaud Schmitt, «The Plot Against America : l'auteur, le lecteur et le complot. », Transatlantica [En ligne], 2 | 2007, mis en ligne le 21 janvier 2008, consulté le 29 avril 2021. URL : http://

journals.openedition.org/transatlantica/1832 ; DOI : https://doi.org/10.4000/transatlantica.1832

Ce document a été généré automatiquement le 29 avril 2021

\section{(c) (i) (9)}

Transatlantica - Revue d'études américaines est mis à disposition selon les termes de la licence Creative Commons Attribution - Pas d'Utilisation Commerciale - Pas de Modification 4.0 International. 


\title{
The Plot Against America : l'auteur, le lecteur et le complot.
}

\author{
Arnaud Schmitt
}

1 Être lecteur de Philip Roth n'est pas une sinécure. La quiétude n'est de toute façon pas ce que le lecteur assidu vient chercher dans cette œuvre intense et protéiforme. Cependant, en fonction du texte qui lui tombe entre les mains, le lecteur de passage ${ }^{1}$ peut s'y sentir mal accueilli. Il serait intéressant d'établir une nomenclature des types de relation qu'un auteur peut entretenir avec son lectorat, mais cette entreprise relèverait de la gageure, et ce pour deux raisons : tout d'abord, certains auteurs ont conscience de s'adresser à un public pluriel et souvent insaisissable et, en second lieu, ces mêmes écrivains sont en général peu diserts sur la question de l'image de leur lectorat, réfutant la possibilité d'un lecteur idéal, même si leurs textes en font le songe. Le lecteur reste un sujet tabou, une matérialité à la fois incontournable mais instable de l'œuvre et celle-ci, comme le souligne parfaitement le philosophe Richard Rorty, est condamnée à attendre que quelqu'un veuille bien la lire pour exister pleinement : « So I should prefer to say that the coherence of the text is not something it has before it is described, any more than the dots had coherence before we connected them " (Rorty in Eco, 97). Roth fait une analyse assez similaire, peut-être un peu moins pragmatique et un peu plus fataliste :

No matter what they say in the classrooms, people read novels and make of them what they make of them [...]. What I write has a particular purpose and use for me, but I've learned that what it means to me isn't what it means to others. When you publish a book, it's the world's book. The world edits it. (Roth in Searles, 110-11)

Ce qui n'empêche pas le même auteur d'être particulièrement exigeant avec son lectorat, de solliciter toute son attention, de le bousculer, de lui mentir même. Roth ne travaille pas exactement dans la congruence. Son mode opératoire est assez simple : briser les évidences, les (ses) automatismes, les (ses) habitudes. Roth a beau faire la constatation citée plus haut quant à l'existence de ses textes dans le monde, il n'empêche qu'au-delà de la pose fataliste se trouve un réel besoin de trouver un interlocuteur prêt à relever le défi. Il ne semble pas excessivement péremptoire de dire que chaque auteur nourrit secrètement le rêve d'une compréhension totale. Cela a été 
remarqué par Umberto Eco ${ }^{2}$ ou encore Tzvetan Todorov ${ }^{3}$. Toute personne s'intéressant au problème de la réception ne peut faire abstraction du mythe du lecteur idéal, ou d'une lecture somme toute parfaite. D'ailleurs, dans une autre déclaration, Roth passe d'une attitude fataliste à une position élitiste, accordant aux seuls auteurs la capacité de comprendre l'intégralité de leurs textes : "The only reading resembling the ideal reading that a writer sometimes yearns for is the writer's reading of himself. Every other reading is something of a surprise [...] a "misreading" " (Roth in Searles, 243-44). Sans vraiment se contredire, Roth trahit ici une position ambivalente vis-à-vis de son lectorat, oscillant entre renoncement et espoir. Cette attitude s'inscrit en filigrane à la fois dans la structure narratologique de son œuvre, et dans le rapport que celle-ci entretient avec le «référentiel», la vie de l'auteur empirique, ce que Jean Ricardou appelle le « bio ». En effet, Roth n'est pas tout à fait innocent dans ce domaine. Si ce que nous pourrions appeler la critique biographique (celle qui cherche dans le texte des clés afin de distinguer la fiction du vécu) lui a mené la vie dure, spécialement après la publication de son texte-scandale Portnoy's Complaint, il a immédiatement repris au lecteur le bâton qu'il avait semblé lui donner afin de faire perdre à celui-ci le goût d'une curiosité de mauvais aloi.

«bio » et provocation

3 Au tournant des années quatre-vingt et quatre-vingt dix, Roth a publié un certain nombre d'ouvrages déclarés paratextuellement comme autobiographiques: The Facts, Patrimony, et Deception, dont le statut générique est tout de même plus trouble.

4 En dehors de ces textes, il est de notoriété publique dans le monde littéraire que Roth a alimenté sa fiction d'expériences personnelles. Cette affirmation peut faire sourire, puisqu'il est difficile pour un auteur de parler d'une autre expérience que de la sienne. Il existe cependant des degrés et des types de proximité différents. Le travail de Thomas Pynchon dans Mason \& Dixon s'articule par exemple autour d'une proximité imaginaire, l'intériorisation et l'appropriation d'un fait historique. L'auteur peut, bien entendu, travailler à partir d'une proximité affective, sans pour autant faire référence à $\mathrm{du}$ « factuel». Lorsque Roth évoque la vie dissolue et tourmentée de son alter ego Nathan Zuckerman, devenu narrateur attitré, après la publication de Carnovsky (texte qui semble, d'après ce qui nous en est dit dans Zuckerman Unbound, très proche thématiquement de Portnoy's Complaint), ou lorsqu'il place dans la trilogie américaine le même Zuckerman dans une maison isolée du Connecticut dans laquelle il mène une existence monacale consacrée à l'écriture (ce qui semble plus ou moins être la situation de Philip Roth depuis les années soixante-dix), il ne fait peut-être pas appel directement à son vécu (comment le savoir?) mais il invite le lecteur qui a une connaissance épitextuelle ${ }^{4}$ de sa vie à établir des parallèles, à tirer des conclusions. Il l'encourage à entremêler sa figure d'auteur et le contenu du texte 5 .

5 Enfin, Roth n'a pas hésité dans Operation Shylock à inclure son double homonymique et, semble-t-il, biographique, dans un texte qui représente sans doute sa plus grande provocation à l'encontre du lecteur. Il promet, en effet, dans une note liminaire, de raconter une « histoire vraie » et, après avoir narré un récit plus que rocambolesque il est vrai, se désavoue et, par la même occasion, désavoue son lecteur (la partie la plus crédule de son lectorat en tout cas).

6 Les intentions autobiographiques de Philip Roth, jusqu'à la publication de The Plot against America, n'ont jamais été univoques. Sa politique architextuelle n'a jamais été d'amener le lecteur à signer un contrat de lecture ouvertement autobiographique, et de 
satisfaire ainsi les lecteurs en quête de référentiel, bien au contraire; et, bien que flirtant à plusieurs reprises avec l'autobiographique, Roth s'est donné beaucoup de mal pour dénoncer tout pacte le condamnant à la véracité et l'éloignant trop longtemps de son passe-temps favori, à savoir la fiction. Dans un texte supposé référentiel comme The Facts, Roth parvient avec une grande aisance à ne visiter que brièvement la sphère autobiographique. Cette entreprise de confession, menée semble-t-il à contrecœur, lui permet, non pas de faire évoluer son discours référentiel, mais bien de redynamiser sa fiction. Dans Deception, le personnage de l'écrivain, qui porte soit dit en passant le nom de Philip Roth, fait la confession suivante : «To compromise some "character" doesn't get me where I want to be. What heats things up is compromising me " (177). Ce précepte créatif établissait déjà le mode opératoire d'Operation Shylock. Et même si un livre comme Patrimony traduit une certaine sincérité de la part de Roth de par sa volonté de faire un travail de deuil de la meilleure manière possible pour un auteur, à savoir textuellement, ce texte ne traite pratiquement pas de son auteur. Le «bio » crée la plupart du temps une strate fictionnelle de plus, permettant à l'auteur de mettre en place la structure d'une fiction dense et complexe. S'inclure en apparence dans son œuvre lui permet aussi, nous l'avons cité plus haut, de continuer à faire ce qu'il sait faire le mieux : travailler dans l'intensité, travailler l'intensité.

7 En soi, cette démarche n'est pas si singulière. Elle est l'apanage de bon nombre d'auteurs postmodernes. Linda Hutcheon, qui a étudié cette mouvance, fait remarquer que la frontière séparant la fiction et la non-fiction, l'art et la vie, a été celle que les auteurs postmodernes ont pris le plus de plaisir à mettre à mal ${ }^{6}$. Cette politique peut s'expliquer par une volonté de transgresser les limites, certes, mais aussi par celle, moins avouée, de donner une leçon à un lectorat jusque-là un peu trop naïf, ou encore trop avide de "faits réels» et venant chercher quelquefois dans la fiction des révélations sur l'auteur empirique. Inutile de rajouter que bon nombre d'auteurs en ont profité pour "faire passer en fraude ${ }^{7}$ leur autobiographie. Les raisons d'une telle démarche sont plurielles, mais tournent souvent autour du plaisir, du besoin que nous avons tous de nous dire et le fait que ce plaisir soit amplifié lorsqu'il se produit dans le cadre d'une publication qui, par la force des choses, multiplie le nombre de nos interlocuteurs.

Le professeur Roth

Depuis des années un aspect séminal de la réception de l'œuvre de Roth s'articule donc autour du rapport hautement problématique entre la volonté de l'auteur de se dire tout en ne se disant pas, et cela sans être vu, et celle du lecteur de le voir dans, et entre, les lignes. Roth a produit des textes suffisamment personnels pour éveiller la curiosité du lecteur tout en le tançant justement pour ses penchants voyeuristes. Ce double discours lui a d'ailleurs souvent valu la foudre des critiques lassés par ce petit jeu, à leur avis, stérile ${ }^{8}$. Difficile de prendre partie dans cette querelle, tant l'œuvre de Roth a atteint des sommets ces dernières années; il est néanmoins difficile d'exonérer totalement l'auteur dont l'innocence sur ce point précis semble loin d'être assurée. On sait que celui-ci a énormément souffert des attaques des adeptes de la critique biographique, notamment à l'occasion de la publication de Portnoy's Complaint. Il lui fut reproché de trahir, à travers ce qui semblait être le récit de sa vie familiale, les secrets de la communauté juive américaine. Bien qu'absurdes, ces attaques furent particulièrement véhémentes, et Roth fut même confronté à des situations extrêmement hostiles, notamment lors de débats en public. Au-delà d'une certaine condescendance que les critiques citées plus haut pensent pouvoir déceler dans l'attitude ambiguë de l'auteur 
vis-à-vis de l'utilisation du "bio » dans sa fiction, nous pouvons aussi deviner une volonté vengeresse de Roth vis-à-vis des plus plébéiens de ses lecteurs. Pour cela, il faut forcément entrer quelque peu dans leur jeu, et nous avons vu plus haut que l'auteur n'hésite pas à miner sa fiction. Certains n'y verront qu'une volonté métafictionnelle, une façon d'amorcer une réflexion sur le thème de l'identité. Cette vision des choses peut être étayée par Patricia Waugh, qui a travaillé sur la métafiction et l'inclusion par l'auteur d'un personnage portant son nom dans sa fiction : "The authors who step into their fictions [...] are locked into a system of endless regress. Their identity disappears the moment that it appears in the fictional text » (142). Cette identite disparait-elle réellement ? Elle est en tout cas évoquée et c'est en soi suffisant. La boîte de pandore est ouverte, et les rapports auteur-lecteur forcément profondément altérés. Ne pas considérer cet aspect des choses équivaudrait à occulter la dimension provocatrice d'une grande partie de la production postmoderne. Il existe après tout d'autres façons d'exercer ses talents métafictionnels, ou de réfléchir avec son lecteur au problème de l'identité.

En résumé, et avant de nous concentrer plus spécifiquement sur le cas de The Plot Against America, et de la nouvelle donne générique proposée, nous pouvons dire que lorsque Philip Roth décide de mêler référentiel et fiction, il semble avoir deux lecteurs ou deux catégories de lecteurs en tête: le lecteur et le Lecteur ${ }^{9}$. Le premier est évidemment celui qui recherche dans la fiction ce qu'il ne devrait pas rechercher (bien que cela y soit tout de même un peu présent) et le second est ce lecteur, tout de même idéal, qui tourne la tête lorsque l'ombre des faits semble soudainement planer sur le texte, ou rit avec l'auteur des mauvais penchants du lecteur empêtré dans ses pulsions voyeuristes.

Les histoires de The Plot Against America

10 Nous avons vu que dans ses prétendues expérimentations autobiographiques, Roth s'adresse à deux types de lecteurs; dans The Plot Against America l'auteur américain reste dans une logique binaire en mêlant deux lignes diégétiques, l'histoire et l'Histoire. Le texte trace dans un premier temps ces deux lignes en parallèle avant de les croiser. Pour résumer très brièvement, l'histoire est celle de la famille Roth au début des années 40 , sa vie quotidienne dans un quartier de Newark, New Jersey; l'Histoire (mais aussi l'intrigue du roman) est plus singulière, puisqu'elle nous compte l'arrivée au pouvoir de l'aviateur Charles A. Lindbergh en 1940, après que celui-ci a battu Franklin Roosevelt aux élections présidentielles. Lindbergh se révèle être un fervent antisémite et décide de se rapprocher de l'Allemagne hitlérienne. Après la mise en place de ces deux lignes diégétiques, Roth nous compte la façon dont la grande histoire va avoir une influence sur la petite, celle d'une famille juive américaine, jusque-là immunisée contre la violence antisémite sévissant par exemple sur le vieux continent. L'interaction est bien évidemment unilatérale. Le modeste destin des Roth n'a aucun impact sur l'impérieux destin de la nation et du monde. L'auteur trouve néanmoins un moyen d'établir une liaison directe entre les deux fabula, par le truchement d'un personnage, le rabbin Bengelsdorf, qui choque toute la communauté juive en décidant de se rapprocher de l'administration Lindbergh. Bengelsdorf a donc à la fois un pied dans l'Histoire, et un autre dans l'histoire, puisqu'il épouse la tante du jeune Philip Roth, Evelyn.

11 Mais la réelle interaction a lieu à un niveau politique, notamment lorsque Lindbergh décide de faire voter une loi, «Homestead 42 ", qui a pour but de disséminer à travers le territoire américain des communautés vivant jusque-là regroupées. Pour les Roth, 
cet endroit se révèle être le Kentucky. La famille devient alors une machine à transformer en tensions internes toutes les secousses qui agitent le pays. Chaque membre est confronté à des choix cornéliens : prendre position, agir en conséquence et, pour les deux fils Roth, Sandy et Philip, assumer le fait d'être en désaccord avec l'autorité suprême, non politique, mais bien parentale. Les doutes soulevés par cette soudaine redéfinition de l'Amérique s'étendent à une autre forme d'appartenance, non plus à la famille cette fois, mais à la communauté. Lorsque l'unité d'un groupe est menacée, chaque individu est amené à définir les valeurs qu'il ou elle associe à ce groupe, et à redéfinir les conditions de son appartenance. Roth consacre une partie de son texte à illustrer ces valeurs à travers la description du quotidien d'un quartier. Pour le narrateur Philip Roth, la judéité ne provient pas de préceptes religieux, mais d'un état socio-historique ${ }^{10}$. Cette vision des choses est assez en accord avec celle de l'auteur Roth, pour qui être Juif coule de source et est un état qui, bien que difficile à définir (Roth se dit avant tout américain ${ }^{11}$ et n'est pas religieux) n'est jamais problématique. Si cela n'est pas le cas pour Zuckerman par exemple, ou la famille Roth dans The Plot Against America, la raison relève avant tout de la dynamique fictionnelle. Roth, en tant qu'écrivain, a pour mode opératoire la mise en tension d'une situation, l'insertion d'un antagonisme : "A character in his predicament is what I have to begin with " (Roth in Searles, 163). Cette version dystopique de l'Histoire américaine s'inscrit parfaitement dans la dynamique diégétique de Philip Roth: transformer un état a priori nonproblématique (être juif aux Etats-Unis) en un état non-congruent (être juif aux EtatsUnis, pays placé imaginairement sous le joug d'un président antisémite). Dans cette logique familière et récurrente, Roth recycle son thème favori : la remise en cause de l'autorité, celle du père plus particulièrement. Car, plus que celle d'un pays, ce que l'auteur nous narre ici est, avant tout, la crise d'une famille, et cela, Roth le fait depuis des années. Le complot dont Roth nous parle est celui qui va chambouler la vie de la famille Roth, de la même manière que la publication de Carnovsky dans Zuckerman Unbound bouleverse la vie de la famille Zuckerman. L'intrigue de The Plot Against America a beau marquer les esprits et déparer dans une œuvre jusque-là plutôt réaliste, il ne peut étouffer cette petite musique du conflit que les fidèles lecteurs de Roth ont l'habitude d'entendre.

12 Malgré le préalable inhabituel pour Roth ${ }^{12}$, The Plot Against America s'inscrit naturellement dans le parcours fictionnel de Philip Roth. La nouveauté ne se situe pas dans la thématique choisie par l'auteur, mais dans la nouvelle donne générique qu'il propose. Cette nouvelle donne entraîne forcément un changement du rapport au lecteur, un aspect de l'œuvre qui nous intéresse tout particulièrement ici.

The Plot Against America et la question du genre

$13 \mathrm{Si}$, comme nous l'avons présupposé, The Plot Against America propose deux lignes diégétiques, l'Histoire et l'histoire, l'identité architextuelle du même texte pourrait être tout aussi clairement délimitée en associant la partie historico-politique à de la fiction, et la chronique familiale à de l'autobiographie. Quels éléments peuvent étayer notre argumentation?

Précisons tout de suite que l'analyse qui va suivre implique un lectorat sinon idéal, mais en tout cas familier de l'œuvre (et de la vie) de l'auteur, puisqu'en fin de compte chaque auteur aspire à cette lecture exhaustive qu'un lecteur fidèle et disponible serait capable d'effectuer. Et même si Rorty précise que chaque lecture n'est qu'un contexte 
supplémentaire dans lequel l'événement du texte se produit ${ }^{13}$, cela n'empêche pas l'auteur, et le lecteur, de rêver à une lecture toujours plus " parfaite ".

S'il ne fait aucun doute que la présidence de Lindbergh relève de la fiction, la description de l'univers familial du narrateur, alors jeune garçon, peut sembler familière à un lecteur fidèle de Roth. Il semblerait donc que l'auteur ait placé une cellule référentielle dans un ensemble fictionnel. Roth nous a plutôt habitués jusque-là à mêler son identité onomastique à un contexte improbable, voire loufoque (Operation Shylock, bien sûr), et à réserver à Zuckerman les touches plus personnelles. La tendance semble se modifier avec The Plot Against America. Non seulement l'identité onomastique du narrateur correspond à celle de l'auteur, mais le lecteur y trouve d'autres « opérateurs d'identification " (Gasparini, 24). Les traits sémantiques de chacun des membres de la famille (père, mère, Sandy et Philip) évoquent ceux que nous connaissons des membres de la famille empirique de l'auteur Philip Roth. Pour établir ce parallèle, le lecteur a dû glaner des informations dans les textes de l'auteur (ceux à statut autobiographique, et ceux qui, sous couvert de la fiction, semblent contenir des "blocs de vécu», la distinction ayant été faite plus haut dans cet article), mais aussi dans la masse d'informations épitextuelles circulant autour d'un auteur de l'importance de Roth ${ }^{14}$. Rappelons grâce à Philippe Gasparini que «l'attribution à un roman d'une dimension autobiographique est donc le fruit d'une hypothèse herméneutique, le résultat d'un acte de lecture " (Gasparini, 32). D'autant plus que, dans le cas de The Plot Against America, nous sommes dans la sphère du "vraisemblable ", et non du "vérifiable " (Gasparini, 30), ce qui est toujours le cas lorsque la confession se limite à un contexte privé, et ne fait aucune allusion à un contexte historique qui pourrait être connu du plus grand nombre. Mais, pour reprendre une distinction faite par Gérard Genette, toute interprétation de texte est de toute façon «transcendante ", la lecture sort le texte de son écrin, celui-ci ne pouvant plus alors prétendre à un statut « immanent » Gasparini souligne avec force que le texte n'est autobiographique que lorsqu'il est perçu ainsi, et même lorsque les évènements évoqués par l'auteur semblent être de notoriété publique.

16 Ainsi, en nous basant sur les écrits personnels de Roth, et sur ce que celui-ci a bien voulu dévoiler de sa vie lors d'entretiens (ou ce qui a été dévoilé par d'autres, voire le cas Claire Bloom), nous (Lecteurs) pouvons décider que l'histoire narrée par l'auteur dans The Plot Against America est en partie autobiographique.

17 S'agit-il pour autant d'autofiction? Il est important de se poser brièvement la question. Des nombreuses définitions données de ce "genre $»^{16}$, aucune ne semble correspondre totalement. Ce texte hybride mêle fiction, autofiction (" une fictionnalisation de soi », selon la définition de Colonna) et autobiographie, si l'on s'en tient aux critères de Lejeune: "récit rétrospectif en prose qu'une personne réelle fait de sa propre existence, lorsqu'elle met l'accent sur sa vie individuelle, en particulier l'histoire de sa personnalité » (Lejeune, 14). De manière concrète, le découpage pourrait se faire ainsi :

Fiction : arrivée au pouvoir de Lindbergh

Autobiographie: références «vraisemblables » à l'histoire familiale de la véritable

famille Roth

Autofiction: l'évolution fictive de la véritable famille Roth dans un contexte historique fictionnel

18 En fin de compte, la partie autofictionnelle représente la zone la plus importante puisque le récit, partant d'une base fictionnelle (l'Histoire) et d'une base autobiographique (l'histoire), évolue principalement dans cet espace inter-générique. 
On sent bien que ce qui intéresse Roth dans ce projet relève justement de cette mixité, en particulier pour un auteur qui a volontiers fait violence à la forme romanesque, afin d'en tirer toutes les possibilités esthétiques, et donc, forcément, sémantiques. Contrairement à ce qui a pu être dit plus haut, le préalable est finalement autobiographique (les souvenirs d'enfance de l'auteur), mais passé au révélateur d'un postulat historique imaginaire. Roth utilise sa famille afin d'imaginer la façon dont celle-ci aurait réagi dans une Amérique atteinte des mêmes maux que ceux dont souffraient certains pays européens.

L'évidence, la clarté

19 Contre toute attente, il ressort finalement de cette alchimie une évidence (formelle, générique, communicationnelle), une quiétude, tant le projet est lisible. Ici, Roth ne se dissimule pas derrière Zuckerman, il ne lance pas non plus un double dans l'arène. Le contenu autobiographique est facilement identifiable par le Lecteur. Pour utiliser une ressource épitextuelle, citons une interview accordée par l'auteur au peu confidentiel magazine Time : " My little rubric that I would recite to myself ... was 'Don't invent it, remember it' [...] People think I have, but I never really have written about my family on the nose before like this $»^{17}$. Bien qu'il faille toujours se méfier des dires d'un auteur en ces temps postmodernes de complot envers le lecteur naïf, les propos de Roth semblent bien en adéquation avec le texte qu'il nous livre. L'évidence et la quiétude formelles et génériques dégagées par ce texte, et ce, malgré le thème anxiogène choisi par l'auteur, proviennent de la facilité à identifier les segments diégétiques et leurs entrecroisements, de l'accessibilité du projet en d'autres termes, accessiblité à laquelle Roth ne nous a pas forcément habitués ${ }^{18}$. Les lecteurs les plus critiques pourront avancer qu'une fois de plus, le texte le plus autobiographique de l'auteur ne se concentre pas sur son moi, mais sur le nous familial, comme Patrimony ne traitait logiquement que du père ${ }^{19}$.

Roth garderait ainsi Zuckerman pour raconter, masqué, sa vie d'adulte et n'aborderait l'autobiographie que de manière oblique, narrant ses proches ou un moi lointain. Nous pouvons opposer deux arguments à cette analyse : 1) la quasi-ubiquité du personnage Zuckerman dans l'œuvre de Roth fait de lui un personnage sémantiquement complexe et dont le rôle n'est depuis longtemps plus limité à évoquer la présence du démiurge ; 2) parler de son adolescence, stade essentiel de la construction d'une identité, et de la cellule familiale consiste à révéler les fondations intimes de sa psyché, car si nous sommes parlés par les autres, pour utiliser une rhétorique lacanienne ${ }^{20}$, notre famille, en particulier, pèse sur notre verbe. Ce dernier élément explique la forte charge référentielle qui semble se dégager de la lecture de The Plot Against America, bien qu'il s'agisse majoritairement de fiction: fiction politique et développement fictif d'un contexte familial.

Un autre espace de réception

21 Au-delà du fait que l'autobiographie est de toute façon un "genre impossible " (Chiantaretto, 9) dont le sujet est par définition fugace, fuyant, voire fictif, en tout cas inexorablement coincé dans un temps à jamais insaisissable selon Ricœur (25), Roth ne cherche pas pour autant ici à se poser en professeur post-moderne mettant en garde le lecteur contre l'illusion du «bio » et l'alertant à la pluralité du moi, comme il a pu le faire avant. Ces ambitions « didactiques» ne disparaissent pas pour autant. Elles sont sûrement inhérentes à la position auctoriale : 
Stories ultimately seek to change the minds and the lives of those they touch. (Benjamin, 67)

Every writer who publishes a novel wants it to be authoritative for her readers, even if authoritatively antiauthoritarian, within the sphere and for the receiving community that the work carves out. (Lanser, 186)

At their best writers change the way readers read. That seems to me the only realistic expectation. (Roth in Searles, 186) autrement et ses objectifs semblent avoir changé. Nous ne sommes plus dans une logique de «dysnarration» (terme emprunté à Robbe-Grillet) évoquée indirectement en début d'article lorsque le Roth des années quatre-vingt et quatre-vingt dix voulait non seulement tancer ses lecteurs voyeuristes mais aussi, à travers la métatextualité et la métafictionnalité de The Counterlife par exemple, montrer l'inaptitude du texte à nommer le réel dans toute sa complexité (ce qui ne l'empêchait pas d'essayer). Sans simplifier outrageusement, nous pourrions avancer que la «dysnarration » est un peu la grande entreprise postmoderne, ce que le déconstructionniste J. Hillis Miller a appelé la fonction « apotropaïque » : «It is a throwing away of what is already thrown away in order to save it " (Hillis Miller, 97). Certes, en débattre avec le lecteur demeure pour Roth une des façons intersubjectives séminales d'établir le contact avec ce dernier ${ }^{22}$. De plus rien n'indique que ce qui a pu sembler être chez lui une vision manichéenne du lectorat ait changé. Il serait à la fois trop simple, trop normatif, voire trop mélioratif de penser que l'auteur se détourne du Lecteur pour tendre la main au lecteur. Cependant, la logique de complot, magnifiée par Operation Shylock, paraît dépassée. Il ne s'agit pas de dire que Roth se soit assagi. En soi, The Plot Against America est à nouveau un pied de nez aux habitudes contractées par le lecteur et l'auteur lui-même. Par son inhabituelle clarté, le projet diégétique de Roth se distingue du reste de sa fiction dans laquelle l'insertion du «bio » est habituellement hautement problématique. Il n'est donc pas question de voir dans The Plot Against America une volonté rédemptrice de la dynamique créatrice rothienne. Ceci est d'ailleurs confirmé par la récente publication de son dernier roman, Everyman, nouvelle variation sur une combinaison thématique récurrente chez Roth depuis la publication de Sabbath's Theater: sexe /déliquescence / mort.

Mais, pour un court moment sur l'axe bibliographico-temporel rothien, Roth baisse la garde et donne à lire à un lecteur, ni idéal, ni trop candide, l'essence de son projet littéraire : "By the time the imagination is finished with a fact, believe me, it bears no resemblance to a fact... Eventually, there's a novel » (Roth in Searles, 220). A l'exception près que The Plot Against America prolonge la description du mythe fondateur, la famille, et lance la fiction sans pour autant totalement effacer les traces du «fait ». Certes, à l'arrivée, il s'agit d'un roman, mais accueillant cette fois du «bio», sans que cela soit problématique. Le conflit est concentré dans la diégèse, les personnages subissent toute 
la charge antagoniste des prémisses fictionnelles de The Plot Against America. Nous ne sommes plus dans une logique de reflets mais de fenêtres. Le lecteur voyeuriste ne court plus après l'image fugace de l'auteur empirique, dont la recherche même est censée lui faire éprouver de la culpabilité; nous voici plutôt dans une logique d'ouverture, ouverture sur un vécu, certes altéré par une idée fictionnelle audacieuse, mais auquel néanmoins on rend hommage. Ce geste réminiscent n'est pas étranger à l'œuvre de Roth, mais il ne s'est jamais auparavant aussi paisiblement associé à sa fiction.

Les ambitions de l'auteur ne sont pas bradées pour autant, le Lecteur n'est pas convié à faire un peu plus de place au lecteur. Cependant en ce qui concerne un aspect précis de son œuvre, le croisement des segments biographiques et fictionnels, Roth réduit l'écart entre les deux. Cette interprétation inexorablement subjective de la façon dont l'auteur s'adresse à son lectorat ne nous mène pas à considérer le geste créateur de Philip Roth comme une main tendue à un lecteur moins exigeant. Comme nous avons pu le souligner, identifier le «bio » dans The Plot Against America reste l'apanage d'un lecteur au fait de l'œuvre rothienne. L'auteur ouvre néanmoins un espace de réception conçu pour un lectorat qui ne voit pas forcément dans le surgissement du référentiel dans la fiction un motif de fuite, de rétractation, de désaveu, de dissimulation, bref de complot. Roth ne brade donc pas pour autant sa fiction, son espace créatif de prédilection, mais accorde une seconde chance au « bio », et à ses adeptes.

\section{BIBLIOGRAPHIE}

Benjamin, Walter, Illuminations (Londres : Fontana, [1968] 1992).

Brooks, Peter, Psychoanalysis and Storytelling (Oxford: Blackwell, 1994).

Colonna, Vincent, L'autofiction. Essai sur la fictionnalisation de soi en littérature, Thèse sous la direction de Gérard Genette, EHESS, 1989, inédite.

Couturier, Maurice, La Figure de l'Auteur (Paris: Editions du Seuil, 1995).

Danziger, Marie A., Text/Countertext, Postmodern Paranoia in Samuel Beckett, Doris Lessing, and Philip Roth (New York : Peter Lang, 1996).

Eco, Umberto, Interpretation and Overinterpretation (Cambridge : Cambrige University Press, 1992).

Epstein, Joseph, « What does Philip Roth want? », Commentary (janvier 1984).

Gasparini, Gérard, Est-il Je ? (Paris : Editions du Seuil, 2004).

Genette, Gérard, Seuils (Paris : Éd. du Seuil, 1987).

-------, Fiction et Diction (Paris : Éd. du Seuil, 1991).

-------, « Peut-on parler d'une critique immanente ? », Poétique 126 (avril 2001), 131-50.

Hillis Miller, J., Ariadne's Thread, Story Lines (New Haven : Yale University Press, 1992).

Hutcheon, Linda, A Poetics of Postmodernism (Londres : Routledge, 1988). 
Lacan, Jacques, Le Séminaire, Livre II : Le Moi dans la Théorie de Freud et dans la Technique de la Psychanalyse (Paris : Éd. du Seuil, 1978).

------, Le Séminaire, Livre XXIII : Le Sinthome (Paris : Éd. du Seuil, 2005).

Lejeune, Philippe, Le pacte autobiographique (Paris : Éd. du Seuil, 1975).

Podhoretz, Norman, « The Adventures of Philip Roth », Commentary (octobre 1998).

------, « Philip Roth : The Great American Novelist », The American Spectator (septembre 1981).

Rabinowitz, Peter J., Before Reading: Narrative Conventions and the Politics of Interpretation

(Columbus : Ohio State University Press, 1988).

Ricoeur, Paul, Temps et Récit, 1 (Paris : Éd. du Seuil, 1983).

Rorty, Richard, « The Pragmatist's Progress », dans Umberto Eco, Interpretation and Overinterpretation (Cambridge : Cambrige University Press, 1992).

Roth, Philip, Zuckerman Bound : A Trilogy [The Ghost Writer (1979), Zuckerman Unbound (1981), The Anatomy Lesson (1984)] and Epilogue [The Prague Orgy (1985)] (Harmondsworth : Penguin, 1989)

-------, The Counterlife (Harmondsworth : Penguin, [1987] 1988).

-------, The Facts : A Novelist's Autobiography (Harmondsworth : Penguin, [1988] 1989).

-------, Deception (Londres : Vintage, 1990).

------, Patrimony : A True Story (Londres : Jonathan Cape, 1991).

-------, Operation Shylock (Londres : Jonathan Cape, 1993).

-------, The Plot against America (New York : Houghton Mifflin Company, 2004).

Salinger, J.D., Raise High the Roof Beam, Carpenters - Seymour : An Introduction (Harmondsworth : Penguin, [1963] 1983).

Searles, Georges J., ed., Conversations with Philip Roth (Jackson : University Press of Mississippi, 1992).

Sniader Lanser, Susan, « Toward a Feminist Poetics of Narrative Voice », dans David H. Richter, ed., Narrative/Theory (White Plains, NY : Longman, 1996).

Todorov, Tzvetan, Mikhail Bakhtine, le principe dialogique (Paris : Editions du Seuil, 1981).

Waugh, Patricia, Metafiction (Londres : Routledge, 1984).

\section{NOTES}

1. Cette expression, « lecteur de passage ", fait bien évidemment référence à la deuxième catégorie de lecteurs mentionnée dans la célèbre épigraphe de Raise High the Roof Beam, Carpenters-Seymour : An Introduction de J.D Salinger : «If there is an amateur reader still left in the world-or anybody who just reads and runs... » (mes italiques).

2. «A text is a device conceived in order to produce its model reader» $(\mathrm{Eco}, 64)$.

3. « S'il n'y a pas de lecteur idéal, qui totaliserait le sens du texte, cela n'empêche pas l'auteur d'y rêver » (Todorov, 170).

4. Dans Seuils, Gérard Genette range sous cette appellation toutes les informations extratextuelles (critiques, études, entretiens...) qu'un lecteur peut glaner sur un auteur et son texte. 
5. Les conséquences sémantiques de l'inclusion de l'auteur dans sa fiction font l'objet d'une analyse intéressante dans le livre de Marie A. Danziger : Text/Countertext, Postmodern Paranoia in Samuel Beckett, Doris Lessing, and Philip Roth.

6. "The most radical boundaries crossed, however, have been those between fiction and non-fiction and-by extension-between art and life » (Hutcheon, 10).

7. L'expression est de Maurice Couturier (213).

8. « Moreover, I was irritated by the literary games Roth took to playing in some of his later, "postmodern" novels. These games consisted in virtually forcing the reader into seeing something as autobiographical and then implicitly rebuking him for doing so (how could anynone be so stupid as not to understand that art "transmutes" reality?) » (Norman Podhoretz, 1998, 33); « Roth has spoken of readers getting a "voyeuristic kick" from reading his autobiography into his books. I think "voyeuristic kick" is exactly the correct phrase, and my first response to it is that, if a writer doesn't wish to supply such kicks, perhaps he would do better not to undress before windows opening onto thoroughfares » (Epstein, 64).

9. La référence à la pensée lacanienne est voulue, notamment bien sûr, la distinction entre autre (imaginaire) et Autre (symbolique) : «L'analyste participe de la nature radicale de l'Autre, en tant qu'il est ce qu'il y a de plus difficilement accessible. Dès lors, et à partir de ce moment, ce qui part de l'imaginaire du moi du sujet s'accorde non pas avec cet autre auquel il est habitué, et qui n'est que son partenaire, celui qui est fait pour entrer dans son jeu, mais avec justement l'Autre radical qui lui est masqué » (Lacan, 1978, 373).

10. "Their being Jews didn't issue from the rabbinate or the synagogue or from their few formal religious practices [...]. Their being Jews didn't even issue from on high [...]. These were Jews who needed no large terms of reference, no profession of faith or doctrinal creed, in order to be Jews [...]. Neither was their being Jews a mishap or a misfortune or an achievement to be "proud" of. What they were was what they couldn't get rid of-what they couldn't even begin to want to get rid of » (The Plot Against America, 220).

11. « To be sure, all three of these writers [Bellow, Malamud, Roth], and especially Bellow, would express resentment when they were identified as Jewish writers, as though this diminished them and made their work less American or less universal » (Podhoretz, 1981, 26).

12. Roth nous a plutôt habitués ces derniers temps à revisiter un contexte historique défini (la guerre du Vietnam dans American Pastoral, le maccarthysme dans I Married a Communist ou le quasi-impeachment de Clinton dans The Human Stain) en tentant d'en embrasser toute la complexité à travers une pluralité de points de vue. Mais il n'est pas étranger pour autant à la fiction politique puisqu'il publia dans les années soixante-dix un brûlot intitulé Our Gang qui s'attaquait avec force et humour à l'administration Nixon.

13. «Each of these supplementary readings simply gives you one more context in which you can place the text-one more grid you can place on top of it or one more paradigm to which to juxtapose it. Neither piece of knowledge tells you anything about the nature of texts or the nature of reading. For neither has a nature » (Rorty in Eco, 105).

14. Même si Roth vit quelque peu en reclus dans sa bâtisse du Connecticut, il se plie cependant aux sacro-saintes interviews qui accompagnent la sortie d'un livre. Certaines d'entre elles ont été rassemblées par George J. Searles dans Conversations with Philip Roth 
(Jackson : University Press of Mississippi, 1992). L'auteur a aussi, malgré lui, fait parler de lui lorsque son ancienne compagne, l'actrice Claire Bloom, a publié un livre à la suite de leur séparation dans lequel elle donne moult détails sur leur vie privée (Leaving a Doll's House. London : Little Brown and Company, 1998).

15. Vues développées dans un article intitulé «Peut-on Parler d'une Critique Immanente? » et publié dans Poétique.

16. Elle peut être vue comme une confession psychanalytique dans un style propre au roman (Doubrosky), comme une « fictionnalisation de soi » (Colonna, 3), ou bien comme le fruit d'un pacte contradictoire du type « Moi, auteur, je vais vous raconter une histoire dont je suis le héros mais qui ne m'est jamais arrivée » (Genette, 1991, 86). 17. Time, 4 octobre 2004, 77.

18. Certains pourront arguer du fait que cette évidence a déjà été amorcée par la trilogie américaine, mais ce serait oublier que cette même trilogie compte un narrateur hautement déclaré et personnalisé en la personne de Nathan Zuckerman, et que dans ce genre de contexte narratif très subjectivisé, les choses ne sont simples qu'en apparence.

19. Vision discutable puisque ce texte traitait de la paternité, et donc d'une relation filspère.

20. « Nous croyons que nous disons ce que nous voulons, mais c'est ce qu'ont voulu les autres, plus particulièrement notre famille, qui nous parle » (Lacan, 2005, 162).

21. Brooks, 49.

22. Dans un des ouvrages de référence de la théorie de la réception Before Reading: Narrative Conventions and the Politics of Interpretation, Peter J. Rabinowitz fait la remarque suivante : « An author has, in most cases, no firm knowledge of the actual readers, who will pick up his or her book. Yet he or she cannot begin to fill up a blank page without making assumptions about the readers' belief, knowledge, and familiarity with conventions [...]. Artistic choices are based upon these assumptions-conscious or unconscious-about readers » (Rabinowitz, 21). Roth se démarque juste par une recherche plus systématique de la confrontation avec les attentes du lecteur. 